\title{
Theoretical study of alkali-atom insertion into small-radius carbon nanotubes to form single-atom chains
}

\author{
Jinlong Yang, ${ }^{1,2}$ H. J. Liu, ${ }^{1}$ and C. T. Chan ${ }^{1}$ \\ ${ }^{1}$ Physics Department, Hong Kong University of Science and Technology, Clear Water Bay, Hong Kong, China \\ ${ }^{2}$ Open Laboratory of Bond Selective Chemistry, University of Science and Technology of China, Hefei, China
}

(Received 10 May 2001; published 8 August 2001)

\begin{abstract}
Using first-principles total energy calculations, we found that the doping of Li into $(6,0)$ nanotubes has no insertion barrier if the tube mouth is passivated. The diffusion barrier is extremely small inside the nanotube, and $\mathrm{Li}$ atoms prefer to reside along the tube axis. The reaction is strongly exothermic. The $\mathrm{Li}-\mathrm{Li}$ interaction inside the nanotube is repulsive but strongly screened. These results point to the possibility of realizing a truly one-dimensional atomic wire inside certain small-radius nanotubes that are fabricated inside zeolite channels if $\mathrm{Li}$ is used as dopant, but other elements like $\mathrm{K}$ would not serve the purpose.
\end{abstract}

DOI: 10.1103/PhysRevB.64.085420

PACS number(s): 71.20.Tx, 61.48.+c, 73.21.-b

Carbon nanotubes have attracted much attention as a beautiful form of nanoscaled material ${ }^{1}$ with possible applications in nanoscaled devices. The possibility of modifying the properties of nanotubes by doping has received a lot of attention. $^{2-5}$ Miyamoto et al. $^{3}$ have considered a chain of $\mathrm{K}$ atoms inside some single-wall carbon nanotubes (SWNT's), and found an exothermic interaction for $(7,0)$ and $(8,0)$ tubules. How the $\mathrm{K}$ atoms can enter the tubules, which is an important issue, was not considered. Although the reaction can be exothermic, SWNT's as a template for a single-atom wire are still elusive unless the insertion barrier is surmountable and if the metal atoms do prefer to sit inside rather than outside the wall. It is not obvious that these conditions can be met. In fact, $a b$ initio molecular dynamics ${ }^{5}$ simulations found large barriers for alkali-atom insertion through the side wall of nanotubes. The calculations of Miyamoto et al. showed that the interstitial sites outside the tubes have lower energies if the tubules form condensed bundles, ${ }^{3}$ and other calculations $^{4}$ for alkali-intercalated nanotube robes found that both exterior and interior sites seem possible.

Recently, SWNT's have been successfully fabricated inside inert $\mathrm{AlPO}_{4}-5$ zeolite channels. ${ }^{6}$ The final products are perfectly aligned monosized SWNT's with extremely small diameters (4-5 $\AA$ ) confined inside the zeolite channels with inner diameters of about $7.3 \AA$. Their existence has been supported by characterization techniques including Raman, x-ray, and direct transmission electron microscopy (TEM) imaging. ${ }^{6}$ These systems are unique as far as doping is concerned since the confinement of the nanotubes inside the zeolite channels naturally prevents the metal atoms from attaching to the outside of the SWNT. The dopants can only be encapsulated. We will see below that atoms inside cannot form clusters, but have to line up as a single-atom wire. These ultrasmall-radius SWNT's thus have some unique structural features that make them ideal platforms for realizing a truly one-dimensional single-atom wire, provided that some necessary conditions can be satisfied. First, the overall atom-tube reaction has to be thermodynamically favorable. Second, the atoms inside the tube should not form small three-dimensional (3D) clusters or 2D patches that are bound to the wall. Third, the diffusion barrier for the atoms inside the tube should be small. Last, and perhaps most importantly, the metal atoms must find their way into the tube. For nanotubes in zeolite channels, this means that the dopant atoms must be able to penetrate the mouth of the nanotube, which is the only location that the outside atoms can enter the interior of these trapped SWNT's.

In the following, we present detailed calculations that show that at least one element $(\mathrm{Li})$ satisfies these conditions, but not all alkali atoms (e.g., K) are equally favorable. The $(6,0)$ tubule will be used as the prototype of these smallradius nanotubes that fits into zeolite channels.

We will first consider the key issues of the insertion barrier and the diffusion barrier inside the tube using the local density functional formalism as implemented in a cluster simulation package ${ }^{7}$ employing an atomic orbital basis set and the Vosko-Wilk-Nusair parametrization of the local exchange-correlation energy. We employ a double-numerical basis set, and the C $2 s$ and C $2 p$ orbitals are represented by two basis functions each, and a $3 d$-type wave function on each carbon atom has been used to describe polarization. The $\mathrm{H}$, Li, and $\mathrm{K}$ atoms are treated in an analogous way. An angular momentum number one greater than the maximum angular momentum number in the atomic orbital basis is applied to specify the multipolar fitting that describes the analytical forms of the charge density and the Coulombic potential. The inner core orbitals are frozen in the calculations to save the computation effort. The atomic geometries are always fully relaxed in our calculations.

The results for Li insertion into the $(6,0)$ tube are summarized in Fig. 1, where we show the energy change as $\mathrm{Li}$ atoms penetrate into the $(6,0)$ nanotube through the mouth of the tube. The reference energy (zero of the binding energy) is that of the total energy of the nanotube and free Li atoms. We used a tubule of finite length to model the $(6,0)$ tubule, and we consider three possible conditions for the mouth of the tube: (i) open, (ii) capped, and (iii) saturated with H. We first consider the case when the tubule is open, with the tubule modeled by $\mathrm{C}_{120}$. Two $\mathrm{Li}$ atoms are arranged to penetrate symmetrically from both ends of the tube. The binding energy as a function of distance measured from the geometric center of the $\mathrm{C}_{120}$ tubule for this case is shown as triangles in Fig. 1. We see that the Li forms a stable carbide at about 


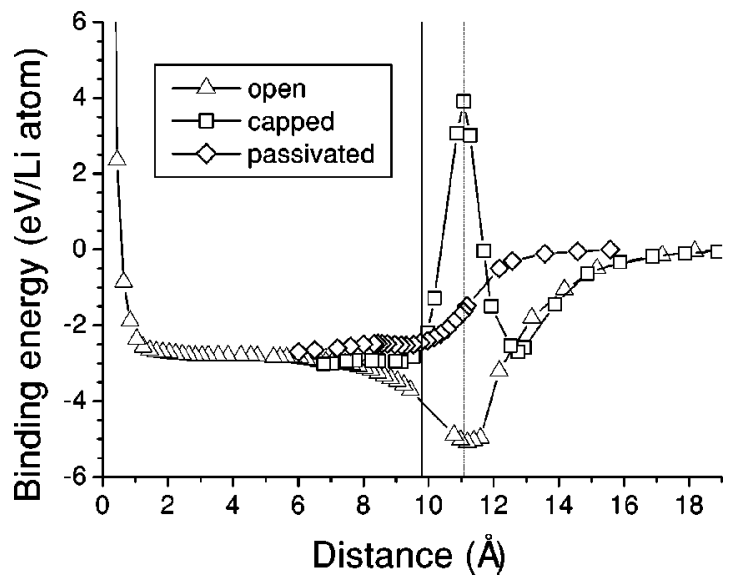

FIG. 1. Binding energy per $\mathrm{Li}$ atom as a function of the distance from the geometric center of the $(6,0)$ tube of a finite length for (i) open tube (triangles), (ii) capped tip (squares), and (iii) tube with atoms in the rim passivated by $\mathrm{H}$ (diamonds).

$1.35 \AA$ outside the geometric edge of the tube, which is marked by the solid vertical line in Fig. 1. Once inside the tubule, $\mathrm{Li}$ atoms are found to have minimum energy along the tube axis (see also Fig. 3 below). The binding energy is very smooth and settles to a rather flat curve when the $\mathrm{Li}$ atom is about $4 \AA$ away from the geometrical rim. There is a difference of approximately $2.3 \mathrm{eV}$ between the interior binding energy and the binding site just outside the mouth of the tube. The smoothness of the curve is essentially due to the small size of the $\mathrm{Li}$ atom, and we will see that the binding energy inside the tube is more structured for $\mathrm{K}$. The flat part of the binding curve (from about 8 to $2 \AA$ ) is actually sloping very gently upwards but shoots up sharply when the $\mathrm{Li}$ atom is about $1.5 \AA$ from the center of the tube. Since we are inserting one $\mathrm{Li}$ atom from each end of the tubule, Fig. 1 can be viewed as the interaction of two $\mathrm{Li}$ atoms in the middle of a strongly polarizable cylinder. Since Li transfers some of its charge to the nanotube, the partially charged $\mathrm{Li}$ ions should repel each other inside the tube. The flat binding curve indicates that the $(6,0)$ nanotube screens the $\mathrm{Li}-\mathrm{Li}$ repulsion very effectively until the $\mathrm{Li}$ atoms are about $3 \AA$ or so away from each other.

We next consider the case of the capped tube, which has an additional hexagonal ring of six $\mathrm{C}$ atoms on the end of $(6,0)$ tip, forming six pentagons with the rim of the tube. The position of the hexagonal cap is marked by the dotted line in Fig. 1. The finite capped $(6,0)$ tube in the calculation has 132 $\mathrm{C}$ atoms. The binding of the $\mathrm{Li}$ as a function of distance for this case is shown in Fig. 1 as squares. There is a local minimum at about $1.65 \AA$ outside the cap of the tip and an energy barrier of nearly $6.6 \mathrm{eV}$ from the bottom of this local minimum ( $3.9 \mathrm{eV}$ from infinity) when the $\mathrm{Li}$ atom penetrates the hexagonal cap. After the cap is penetrated, the energy of the $\mathrm{Li}$ in the tube becomes essentially the same as the other case.

The diamond symbols in Fig. 1 represent the binding energy when the rim of the nanotube is passivated by $\mathrm{H}$ (modeled by $\mathrm{C}_{120} \mathrm{H}_{12}$ ). In this case, the energy of the $\mathrm{Li}$ atom decreases monotonically as it approaches and penetrates the tip. There is no energy barrier, and the polarizable nanotube "sucks" the $\mathrm{Li}$ atoms into the tube. Capillary suction in nanotubes was first predicted by theory ${ }^{8}$ and observed experimentally for the filling multiwalled tubes with lead nanowire. ${ }^{9}$

Summarizing, we see that the chances for Li atoms being successfully doped into the tube depends on the condition of the mouth. There is an insertion barrier if the tube mouth is capped. If the tube is open but unsaturated, the global minimum is a Li carbide state outside the tube. However, Li will be sucked into the tube if the tube is open with the edge atoms passivated since there is no insertion barrier and the chemical potential is substantially lower inside the tube than outside. The local density approximation (LDA) binding energy relative to a free $\mathrm{Li}$ atom is about $2.8 \mathrm{eV}$. If we use generalized gradient approximation (GGA) (Ref. 10) instead, which usually cures the overbinding of LDA calculations, the binding energy is found to be $2.1 \mathrm{eV}$ compared with the free atom. This is about $0.5 \mathrm{eV}$ lower than the experimental cohesive energy of $1.6 \mathrm{eV}$ in bulk $\mathrm{Li}$, and thus the reaction is exothermic. We thus conclude that if the tube is open, $\mathrm{Li}$ atoms can find its way into these small radius tubules and the $\mathrm{Li}$ atoms prefer to sit along the axis of the tubules.

The form of the energy curve in Fig. 1, which gives both the Li-tube interaction and the screened $\mathrm{Li}$ - $\mathrm{Li}$ interaction when the two $\mathrm{Li}$ atoms are close together, has interesting implications. The smoothness of the Li-C binding energy curve ensures that there is no docking of the $\mathrm{Li}$ to some particular positions of the tube. At room or elevated temperatures, $\mathrm{Li}$ atoms should be able to diffuse essentially freely along the axis of the tube, making way for other $\mathrm{Li}$ to enter the tube. The $\mathrm{Li}-\mathrm{Li}$ interaction is also weakly repulsive at long distances and thus newly arriving $\mathrm{Li}$ atoms will push the other $\mathrm{Li}$ atoms into the interior while the strongly repulsive interaction at close distances ensures that $\mathrm{Li}$ will not form clusters inside the tube. These facilitate the formation of a truly one-dimensional atomic wire.

Up to this point, we considered a dilute limit. To see whether a high concentration of $\mathrm{Li}$ can exist inside the tube, we calculate the differential heat of formation of $\mathrm{Li}$ atoms by putting up to nine $\mathrm{Li}$ atoms one by one into the $(6,0)$ tube, modeled by the $\mathrm{C}_{120} \mathrm{H}_{12}$ configuration. The differential heat of formation, plotted in Fig. 2, is defined as $\mathrm{H}_{n}=-E\left(\mathrm{Li}_{n}\right.$ + tube $)+E\left(\mathrm{Li}_{n-1}+\right.$ tube $)+E_{L i}$, where $E\left(\mathrm{Li}_{n}+\right.$ tube $)$ is the total energy of the finite-sized nanotube with $n \mathrm{Li}$ atoms inside, atomic coordinates optimized. It measures the energy gain when an extra $\mathrm{Li}$ atom enters the nanotube that already has $n-1 \mathrm{Li}$ atoms inside. We see from Fig. 2 that the energy is exothermic relative to an isolated $\mathrm{Li}$ atom all the way up to nine $\mathrm{Li}$ atoms. As the number of $\mathrm{Li}$ atoms inside increases, there is an overall decrease in the differential heat of formation, a consequence of the repulsive $\mathrm{Li}-\mathrm{Li}$ interactions. On top of the overall decrease, we see an even-odd "quantumsize" oscillation typical that of a short linear chain. The maximum doping concentration can in principle be deduced if we know the chemical potential of the Li outside the tube. What is more important is that for $n$ up to 5 , there is little 


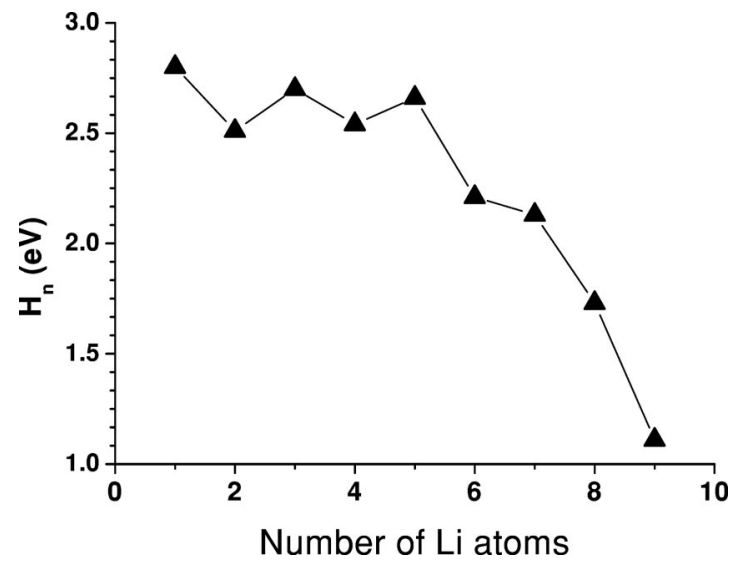

FIG. 2. The differential heat of formation as an increasing number of $\mathrm{Li}$ atoms are doped into the interior of the tube.

change of $\mathrm{H}_{n}$ except for finite-size oscillations. This shows that a large quantity of Li can be doped into the tube in an exothermic manner.

While the real-space cluster technique is best suited for considering the dopant insertion, the $k$-space method with periodic boundary condition is more efficient for higher concentrations. We used a plane-wave pseudopotential formulation ${ }^{11}$ within the framework of the GGA. ${ }^{10}$ We used ultrasoft pseudopotentials for $\mathrm{C}$ and $\mathrm{Li}$ and a plane-wave cutoff of $26.3 \mathrm{Ry}$. We used a supercell geometry ${ }^{12}$ in which the tubules are aligned in a hexagonal array with a lattice constant of $11 \AA$ so that the shortest distance between the adjacent tube atoms is about $6 \AA$. We considered three concentrations with nominal formulas $\mathrm{C}_{12} \mathrm{Li}, \mathrm{C}_{24} \mathrm{Li}$, and $\mathrm{C}_{48} \mathrm{Li}$, with Li-Li distances at $2.13,4.25$, and $8.51 \AA$, respectively. $K$ points are sampled on a uniform $1 \times 1 \times 20$ grid for $\mathrm{C}_{12} \mathrm{Li}$, and equivalent $k$-point sets are used for other concentrations. We calculated the heat of formation relative to bulk bcc Li and a fully relaxed tube. We show in Fig. 3 the energy change as we move the Li atom from the axis out towards the center of one of the hexagons on the sidewall. This single figure contains several pieces of information. First, it shows clearly that the center of the tube is the lowest-energy position. The $\mathrm{Li}$ atoms do like to form a single-atom chain along

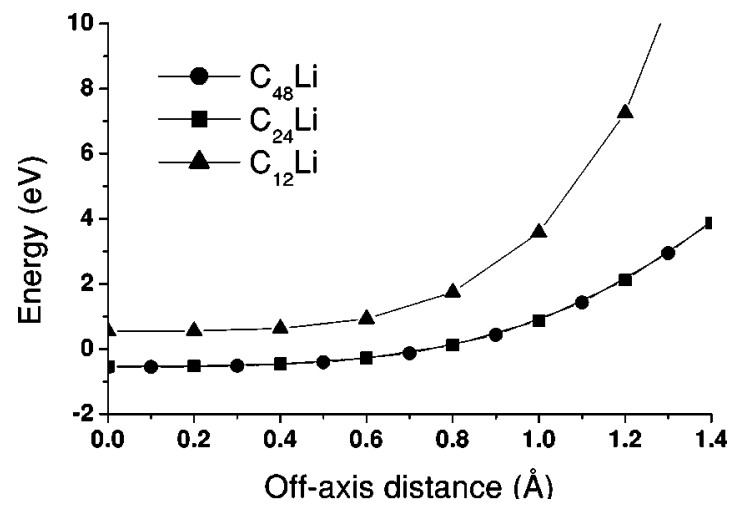

FIG. 3. The energy change as we move the Li atom out from the tube axis radially towards the center of a hexagonal ring for $\mathrm{C}_{12} \mathrm{Li}$, $\mathrm{C}_{24} \mathrm{Li}$, and $\mathrm{C}_{48} \mathrm{Li}$.

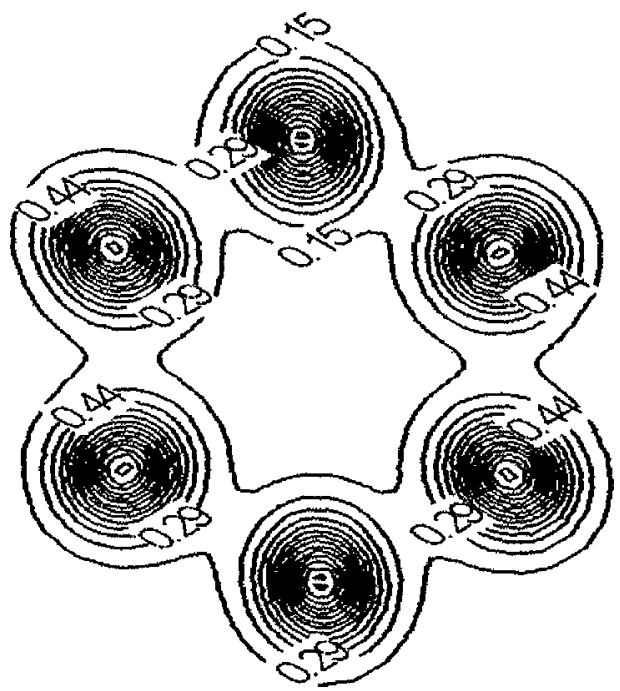

FIG. 4. The (pseudo)charge density contour plot on a plane perpendicular to the tube axis, cutting through a $\mathrm{Li}$ atom on the tube axis. The tube has a nominal composition of $\mathrm{C}_{24} \mathrm{Li}$. The contours are in units of electrons $/ \AA^{3}$. On a much finer scale, one sees that the charge density of carbon atoms is polarized towards the axis of the tube.

the axis, rather than attaching themselves to the wall. Second, the energy curves of $\mathrm{C}_{24} \mathrm{Li}$ and $\mathrm{C}_{48} \mathrm{Li}$ literally coincide with each other, while that of $\mathrm{C}_{12} \mathrm{Li}$ has a higher energy. This reinforces that notion that the $\mathrm{Li}-\mathrm{Li}$ interaction is indeed well screened and becomes noticeably repulsive only when the $\mathrm{Li}-\mathrm{Li}$ distance is under $3 \AA$. Third, the heat of formation for $\mathrm{C}_{24} \mathrm{Li}$ is $0.54 \mathrm{eV}$ relative to bulk $\mathrm{Li}$, implying that the reaction is strongly exothermic. We have also calculated the energy as a function of $\mathrm{Li}$ positions for $\mathrm{C}_{24} \mathrm{Li}$ along the tube axis, and found that the sliding barrier (with the whole chain moving together) is very small, in the order of $0.01 \mathrm{eV}$. These periodic unit cell results corroborate with the real space calculations. The density of states (DOS) at the Fermi level for $\mathrm{C}_{24} \mathrm{Li}$ are 0.13 states/eV/(carbon atom), about twice that of the value 0.07 we found for pure $(6,0)$. This higher density of states, together with possible stronger electronphonon coupling due to the large curvature of these small radius tubes, makes them plausible candidates for observing superconductivity. ${ }^{3}$ Figure 4 shows a (pseudo)charge density contour plot for $\mathrm{C}_{24} \mathrm{Li}$ on a plane perpendicular to the tube axis, cutting through the Li atom in the middle of the tube. There is a significant charge transfer between $\mathrm{Li}$ and the C-tube wall. We can hardly see any valence charge remaining in the Li site. Judging from the contour plot, essentially all the $2 s$ electrons of the $\mathrm{Li}$ are donated to the carbon wall.

We have also considered tubes like $(5,0)$ with a smaller radius (diameter $4.05 \AA$ ). The qualitative results remain the same. For the $(5,0)$ tube the reaction is even more exothermic (heat of formation relative to bulk $\mathrm{Li}$ is $0.72 \mathrm{eV}$ for $\mathrm{C}_{20} \mathrm{Li}$ ). There is no penetration barrier if the tube mouth is passivated and the sliding diffusion barrier is about $0.16 \mathrm{eV}$, which is still fairly small.

We now consider the doping of the $(6,0)$ with $\mathrm{K}$, which has a larger atomic size. The binding energy as a function of 


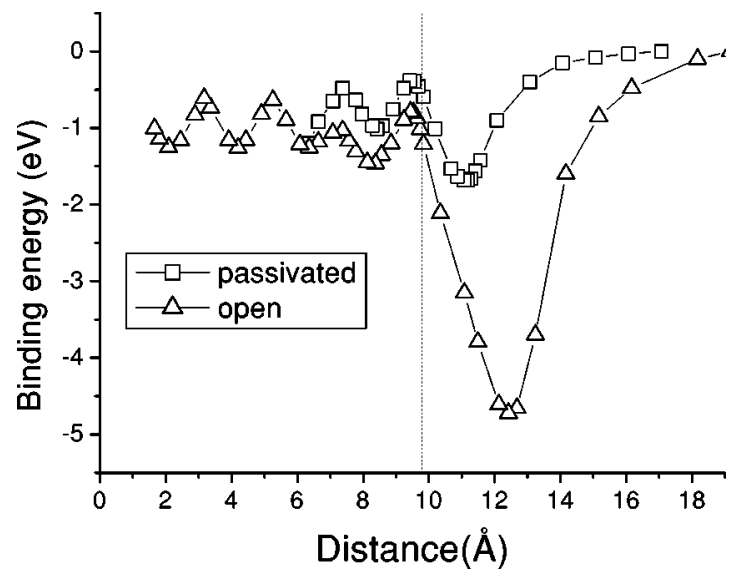

FIG. 5. Binding energy per $\mathrm{K}$ atom as a function of the distance from the geometric center of the $(6,0)$ tube of a finite length for (i) truncated tube (triangles) and (ii) tube with the atoms on the rim passivated with $\mathrm{H}$ (squares).

distance from the geometric center of the tube is shown in Fig. 5. As in the case of Li, the binding energy is calculated with a $\mathrm{C}_{120}$ tubule and two $\mathrm{K}$ atoms, each approaching from one end of the tube. For the case of a truncated tube with open, unsaturated tip, the binding energies are marked by triangles. We find a deep minimum with binding energy of about $4.7 \mathrm{eV}$ when the $\mathrm{K}$ atom is about $2.6 \AA$ outside the geometric rim of the tube, which is marked by the vertical dotted line in the figure. When the $\mathrm{K}$ atom penetrates into the interior of the tube, it prefers to reside on the axis. We see oscillatory behavior in the binding-energy, with the lowestenergy, position near the center of the hexagonal rings and the highest-energy position at the zigzag ring of $\mathrm{C}$ atoms, and an energy difference of over $0.65 \mathrm{eV}$ between the two sites so that there is a noticeable barrier for the $\mathrm{K}$ atoms to diffuse in the interior. As in the case of $\mathrm{Li}$, the Coulombic repulsion between the pair of $\mathrm{K}$ atoms inside the tube is strongly screened. If the tube mouth is passivated with $\mathrm{H}$ atoms, the binding energy is shown by the squares. The $\mathrm{K}$ atom has a binding site at about $1.3 \AA$ from the geometric edge of the tube. When the $\mathrm{K}$ atom penetrates into the tube, it shows the same oscillatory behavior in the binding energy as the previous case, except that the energies are higher, and the two binding energy curves merge together when the $\mathrm{K}$ atom is about $4 \AA$ deep from the tip. We have also calculated the binding energies when the $\mathrm{K}$ atoms penetrate the capped tube, modeled by the $\mathrm{C}_{132}$. There is a huge barrier of about $20 \mathrm{eV}$ for the $\mathrm{K}$ atom to penetrate the hexagonal cap. Since the energy scale is so different, the energy curve is not included in Fig. 5. We note that if we repeat the calculation with the GGA, the binding energy of the $\mathrm{K}$ atom is actually positive (about $0.2 \mathrm{eV}$ at the lowest point), implying that the $\mathrm{K}$ atom has a higher energy inside the tube than outside the tube. The fairly big diffusion barrier together with the unfavorable binding energy implies that doping $\mathrm{K}$ into these small tubes would not be favorable.

For completeness, we also considered the case of $\mathrm{K}$ atoms doping into the $(8,0)$ tube with a passivated rim, modeled by a tube of finite length with $128 \mathrm{C}$ atoms and $8 \mathrm{H}$ atoms on each rim. We found that the behavior is very similar to that of $\mathrm{Li}$ atoms doping into a passivated $(6,0)$ tube. More specifically, we see no insertion energy barrier, no local minimum outside the tube, and the binding energy decreases monotonically from outside to inside. The binding energy curve is fairly smooth when the $\mathrm{K}$ atoms are inside. The $\mathrm{K}-\mathrm{K}$ interaction is strongly screened by the $(8,0)$ tube, and there is no significant docking effect. These results show that the difference between $\mathrm{Li}$ and $\mathrm{K}$ we observed for the $(6,0)$ tube in the previous section is essentially due to the size difference. But of course the nanotubes inside zeolite channels have a maximum radius which cannot be adjusted to fit the dopant, and we can only vary the dopant to fit into tube.

In conclusion, we found that $\mathrm{Li}$ prefers to occupy sites along the tube axis in $(6,0)$ and $(5,0)$ carbon nanotubes and the interaction is exothermic. If the atoms at the rim can be passivated, there is no insertion barrier and there is literally no barrier for Li to diffuse inside. It is thus thermodynamically and kinetically favorable for $\mathrm{Li}$ to form a single line of atoms inside small-diameter $(4-5 \AA)$ nanotubes that are fabricated and confined inside inert zeolite channels. If realized experimentally, we would have an interesting system in which zeolite crystals form a template for nanotubes which in turn serve as a template for one-dimensional wires.

We thank Professor P. Sheng and Dr. Z.K. Tang for many discussions. This work was supported by RGC-HK through Grant No. HKUST6128/98P. Y.J. was also supported by NSFC and NKBRSF(G1999075305). The pseudopotential calculations were performed using VASP (Ref. 11) (Vienna $a b$ initio simulation program) developed at the Institut für Theoretische Physik of the Technische Universität Wien, and we thank Dr. G. Kresse for helpful information.
${ }^{1}$ S. Iijima, Nature (London) 354, 56 (1991).

${ }^{2}$ See, e.g., A.S. Claye, N.M. Nemes, A. Jnossy, and J.E. Fischer, Phys. Rev. B 62, R4845 (2000); B. Ruzicka, L. Degiorgi, R. Gaal, L. Thien-Nga, R. Bacsa, J.-P. Salvetat, and L. Forro, ibid. 61, R2468 (2000).

${ }^{3}$ Y. Miyamoto, A. Rubio, X. Blase, M.L. Cohen, and S.G. Louie, Phys. Rev. Lett. 74, 2993 (1995).

${ }^{4}$ J. Zhao, A. Buldum, J. Han, and J.P. Lu, Phys. Rev. Lett. 85, 1706
(2000); G. Gao, T. Cagin, and W.A. Goddard III, ibid. 80, 5556 (1998).

${ }^{5}$ A.A. Farajian, K. Ohno, K. Esfarjani, Y. Maruyama, and Y. Kawazoe, J. Chem. Phys. 111, 2164 (1999).

${ }^{6}$ Z.K. Tang, H.D. Sun, J. Wang, J.S. Chen, and G.D. Li, Appl. Phys. Lett. 73, 2287 (1998); H.D. Sun, Z.K. Tang, J.S. Chen, and G.D. Li, Solid State Commun. 109, 365 (1999); H.D. Sun, Z.K. Tang, J.S. Chen, and G.D. Li, Appl. Phys. A: Mater. Sci. 
Process. 69, 381 (1999); N. Wang, Z.K. Tang, G.D. Li, and J.S. Chen, Nature (London) 408, 51 (2000).

${ }^{7}$ DMOL is a registered software package from Molecular Simulations Inc.

${ }^{8}$ M.R. Pederson and J.Q. Broughton, Phys. Rev. Lett. 69, 2689 (1992).

${ }^{9}$ P.M. Ajayan and S. Iijima, Nature (London) 361, 333 (1993).
${ }^{10}$ J.P. Perdew and Y. Wang, Phys. Rev. B 45, 13244 (1992).

${ }^{11}$ See G. Kresse and J. Hafner, Phys. Rev. B 47, 558 (1993); 49, 14251 (1994); G. Kresse and J. Furthmüller, Comput. Mater. Sci. 6, 15 (1996).

${ }^{12}$ X. Blase, L.X. Benedict, E.L. Shirley, and S.G. Louie, Phys. Rev. Lett. 72, 1878 (1994). 